\title{
Time-resolved Laser Fluorescence Spectroscopy of Organic Ligands by Europium : Fluorescence Quenching and Lifetime Properties
}

\author{
A. Nouhi ${ }^{\mathrm{a}, *}$, H. Hajjoul ${ }^{\mathrm{a}}$, R. Redon ${ }^{\mathrm{a}}$, J.P. Gagné ${ }^{\mathrm{b}}$, S. Mounier ${ }^{\mathrm{a}}$ \\ ${ }^{a}$ Laboratoire PROTEE, Université de Toulon, CS 60584-83041 Toulon Cedex 9, France \\ ${ }^{b}$ Institut des sciences de la mer de Rimouski, Université du Québec, Canada
}

\begin{abstract}
Time-resolved Laser Fluorescence Spectroscopy (TRLFS) has proved its usefulness in the fields of biophysics, life science and geochemistry to characterize the fluorescence probe molecule with its chemical environment. The purpose of this study is to demonstrate the applicability of this powerful technique combined with Steady-State (S-S) measurements. A multi-mode factor analysis, in particular $\mathrm{CP} / \mathrm{PARAFAC}$, was used to analyze the interaction between $\mathrm{Eu}-$ ropium (Eu) and Humic substances (HSs) extracted from Saint Lawrence Estuary in Canada. The Saint Lawrence system is a semi-enclosed water stream with connections to the Atlantic Ocean and is an excellent natural laboratory. $\mathrm{CP} / \mathrm{PARAFAC}$ applied to fluorescence S-S data allows introspecting ligandsmetal interactions and the one-site 1:1 modeling gives information about the stability constants. From the spectral signatures and decay lifetimes data given by TRLFS, one can deduce the fluorescence quenching which modifies the fluorescence and discuss its mechanisms. Results indicated a relatively strong binding ability between europium and humic substances samples (Log $\mathrm{K}$ value varies from 3.38 to 5.08 at $\mathrm{pH}$ 7.00). Using the Stern-Volmer plot, it has been concluded that static and dynamic quenching takes places in the case of salicylic acid and europium interaction while for HSs interaction only a static quenching
\end{abstract}

\footnotetext{
* Corresponding author

Email address: nouhi@univ-tln.fr (A. Nouhi )
}

Preprint submitted to Journal of ${ }^{A} T_{E} X$ Templates

November 13, 2017 
is observed.

Keywords: Fluorescence quenching, Europium (III), Natural Organic Matter, Time-resolved laser-induced fluorescence spectroscopy, Parallel Factor Analysis, Lifetime Analysis

\section{Introduction}

Improved insight on the interactions between natural dissolved organic matter with lanthanides and actinides has been widely studied Kim et al. (1989); Moulin et al. (1991); Kim et al. (1993); Panak et al. (1996); Czerwinski et al.

5 (1996); Tiseanu et al. (1998); Morgenstern et al. (2000); Monsallier et al. (2003); Schmeide et al. (2006); Brevet et al. (2009); Jain et al. (2009); Lukman et al. (2012); Pacold et al. (2014). Indeed, it is of paramount importance to better understand transport and toxicity pathways of metal ions as well as creating efficient remediation techniques for contaminated environments Hiraide (1992); Dube et al. (2001); Choppin (2007); Sachs and Bernhard (2011); Cobelo-García et al. (2015). Various spectroscopic techniques are used for these purposes. Fluorescence spectroscopy in S-S measurements provides quantitative and qualitative information. However, more data such as fluorescence lifetime measurements could be gathered using TRLFS. In fact, TRLFS is a convenient technique when dealing with different elements in the same sample because it can offer the spectral and temporal resolution together with its high sensitivity. The discriminating capability of TRLFS relies on the fact that the different chemical species between organic matter and a fluorescent metal ion can have different decay lifetimes and spectral shapes which is adequate for the study of such a 20 complex system Collins et al. (2011). The greatest advantages of TRLFS are its relatively low detection limit and good discriminating capability for different chemical species.

An assessment of the influence of HSs on the mobility of Eu in the environment is necessary and has to include knowledge of stability constants for the formation

of $\operatorname{Ln}(\mathrm{III})$-HSs complexes. In this work, complexation of $\mathrm{Eu}^{3+}$ by Humic Acids 
(HA) and Fulvic Acids (FA) from St. Lawrence Estuary (Canada) was first studied by fluorescence quenching using Excitation Emission Matrices of fluorescence (EEMs) with a large range of europium concentration. Metal additions were performed using logarithmic increments to cover a wide range of concentration from

30 $2.2 \times 10^{-7}$ to $1.5 \times 10^{-3} \mathrm{M}$. The obtained data was treated by CP/PARAFAC to extract the different fluorescent components using a MATLAB ${ }^{\circledR}$ home-made program Bro (1997); Luciani et al. (2009). Then a fitting based on the onesite model (1:1) Ryan and Weber (1982) was done on the CP/PARAFAC components to determine their stability constants. TRLFS is a complementary technique to determine the composition of the mixtures by measuring the fluorescence lifetimes and see its variation with the metal addition. Fluorescence properties of $\mathrm{Eu}(\mathrm{III})$ with Salicylic Acid (SA) and HSs complexes were studied from $\mathrm{Eu}(\mathrm{III})$ site Irving and Sinha (1970); Hasegawa et al. (1989); Tiseanu et al. (1998); Hasegawa et al. (1990); Toraishi et al. (2005); Brevet et al. (2009); Jain

40 et al. (2009); Kuke et al. (2010); Lukman et al. (2012); Saito et al. (2017) and also from the ligand site, but only for SA Aoyagi et al. (2004). Analysis of the literature has shown that data of humic substances complexes with $\mathrm{Eu}(\mathrm{III})$ from the ligand site is still lacking. As a matter of fact, it raises some interesting questions that have not been discussed before. Therefore, with this study, we wanted to investigate how these interactions occur focusing on the HSs fluorescence emission. Analysis of fluorescence lifetimes and Stern-Volmer plots Lakowicz (2006) will give reliable information on the quenching process that takes place. This line of study is important to settle a general relationship between organic matter properties from St Lawrence Estuary and static/dynamic quenching contributions.

\section{Experimental setup}

\subsection{Sample preparation}

The complexation of $\mathrm{Eu}(\mathrm{III})$ by $\mathrm{SA}$ was first studied in order to calibrate our assays and compare our results with literature Aoyagi et al. (2004). Indeed, 
the compositions of HSs are complex and partly unknown. The HSs are composed of a complex mixture of organic and organomineral compounds and can differ depending on its type. Therefore, SA provides a carboxyl and hydroxyl group that mimic the important functionalities of HSs Stevenson (1994) and form strong complexes with $\mathrm{Eu}(\mathrm{III})$ Kuke et al. (2010).

${ }_{60}$ Salicylic acid (NormaPur ${ }^{\circledR}$, Prolabo) was used as received. Its aqueous solution was prepared at a concentration of $5 \times 10^{-4} \mathrm{M}$ with UHQ water. HA and FA were obtained from the St. Lawrence Estuary Tremblay and Gagné (2009) following the IHSS protocol Swift (1996).

Europium standard (1,000 mg.L $\mathrm{L}^{-1}$ in $2 \% \mathrm{HNO}_{3}$, SPEX CertiPrep) was used as 65 a quencher without further purification.

Twelve solutions with different concentrations of $\mathrm{Eu}(\mathrm{III})$ were prepared by mixing the stock solutions in a $1 \mathrm{~cm}$ quartz cell. The final concentrations of SA, HA and FA were kept constant at respectively $5 \times 10^{-5} \mathrm{M}, 11$ and $16 \mathrm{mg}$ of matter per liter. Eu(III) was added using logarithmic increments of concentrations from $2.2 \times 10^{-7} \mathrm{M}$ to $1.5 \times 10^{-3} \mathrm{M}$. Every sample was allowed to equilibrate for at least $15 \mathrm{~min}$ and no precipitation was observed at higher europium concentration. The $\mathrm{pH}$ of the samples was adjusted to $7.00 \pm 0.09$ using $1 \mathrm{~mL}$ of HEPES buffer $(0.1 \mathrm{M})$. HEPES was used to maintain the $\mathrm{pH}$ constant and avoid $\mathrm{pH}$ adjustment for each sample. Preliminary measurements without metal additions

75 were done to verify that there is no interaction between the fluorescent ligands and the HEPES buffer. The results did not show any variation of the fluorescence intensity. Thus, no interaction between the HEPES buffer and ligands was observed. A total volume of $3 \mathrm{~mL}$ was achieved by adding UHQ water. Necessary $\mathrm{pH}$ adjustments of metal solutions were done with $\mathrm{NaOH}(1 \mathrm{M})$ and $\mathrm{HCl}(1 \mathrm{M})$ and the changes in the volume were taken into account.

\subsection{Fluorescence and lifetime measurements}

Two series of experiments were done on the prepared samples. In order to perform S-S measurements, a spectrophotometer (HITACHI F4500) equipped with a Xenon lamp $(450 \mathrm{~W})$ and connected to a computer was used. Twelve 
individual EEMs gathered from S-S measurements of SA, HA, and FA were collected in fluorescence quenching experiments. The excitation wavelength range was from $250 \mathrm{~nm}$ to $450 \mathrm{~nm}$ with a $10 \mathrm{~nm}$ slit and $5 \mathrm{~nm}$ excitation step. The corresponding emission wavelengths were collected at $5 \mathrm{~nm}$ step between 300 $\mathrm{nm}$ and $700 \mathrm{~nm}$ and $10 \mathrm{~nm}$ slit. The scan speed was set at 2,400 nm. $\mathrm{min}^{-1}$ and 90 the photomultiplier tension was at $700 \mathrm{~V}$.

TRLFS data was carried out using a pulsed Nd:YAG laser (Quanta-Ray INDI, Spectra Physics) and performed at room temperature (294 K). The Nd:YAG source provides an excitation with a pulse width of $5 \mathrm{~ns}$ and a repetition rate of $20 \mathrm{~Hz}$. The excitation source for TRLFS was the third harmonic generation ${ }_{95}(355 \mathrm{~nm})$ and the fluorescence emission was collected at 90 degrees through a bundle made of 16 optical fibers of $200 \mu \mathrm{m}$ into the entrance slit of a CzernyTurner spectrograph (TRIAX 180, Horiba Jobin-Yvon). The latter has three UV-VIS gratings $(300 \mathrm{~g} / \mathrm{mm}, 900 \mathrm{~g} / \mathrm{mm}$ and 2,400 g/mm). The spectral resolutions were respectively $0.27 \mathrm{~nm}, 0.09 \mathrm{~nm}$ and $0.034 \mathrm{~nm}$. The second grating was used for these experiments.

The fluorescence signal was detected by an intensified couple charged device camera (ICCD 300V, Horiba Jobin-Yvon). For the time-resolved measurements, the initial timing of the fluorescence signal was varied by changing the delay of the trigger through a pulse generator (DG535, Stanford Research System). In order to increase the accuracy of the measurements, each laser pulse was corrected in energy fluctuation by splitting the laser beam through a pyroelectric sensor (PE10, Ophir Electronics). The power of pulse was constant within around 3-5 $\mu \mathrm{J}$ pulse during the acquisition. Seven hundred spectra were accumulated at each time step of $0.2 \mathrm{~ns}$ and a time delay of $200 \mathrm{~ns}$ with a $35 \mathrm{~ns}$ gate width. These parameters were set to measure the fluorescence focusing on the ligand fluorescence emission.

Every mixture was allowed to equilibrate for at least 10 min and bubbled by a nitrogen flux to avoid photobleaching. 


\subsection{Data Treatment}

the contribution of components to the EEMs by separating independent sources after elimination of scattering phenomena Bro (1997). The first step of decomposition consists of cleaning all EEMs from the diffusion signals (Rayleigh and Raman) by cutting the Rayleigh diffusion bands (20 nm) and applying the Zepp procedure Zepp et al. (2004). No inner effect was encountered because chosen concentrations were in the linear zone of fluorescence. Dilution curves were done to determine these value (data not shown). Thus, no numerical correction was applied to the EEMs. The estimation of the number of components is particularly critical because users have to test a range of model to evaluate the best number of components. In this study, it was defined by evaluating the CORCONDIA score. Decomposition investigation was done from 1 to 4 components and the number of component giving a CORCONDIA score higher than $60 \%$ was selected as optimal model. Iterations and number of runs were implemented until the algorithm converged.

130 The optimization between experimental and calculated fluorescence using onesite model with one ligand Ryan and Weber (1982) was executed by evaluating the bias errors. The bias is equal to the sum of the square of the subtraction between the experimental fluorescence intensity and the calculated fluorescence intensity.

${ }_{35}$ Data obtained with TRLFS were analyzed using a home-made MATLAB ${ }^{\circledR}$ program. The evaluation of the best fitting of the integrated fluorescence intensity was done by time deconvolution to determine fluorescence amplitudes and fluorescence lifetimes. Supposing a system excited by an infinitely sharp pulse of light ( $\delta$-function) and assuming the response to be linear, the measured fluorescence decay is given by the convolution integral :

$$
y(n)=h(n) \otimes I R F
$$

where $h(n)$ is the intrinsic fluorescence decay and IRF is the Instrument Response Function. Experimentally, the IRF is gathered by measuring only the 
solvent diffusion.

The estimation of $\mathrm{h}(\mathrm{n})$ was achieved by time deconvolution in order to obtain

the amplitudes of each fluorophore and lifetimes. Assuming a multi-exponential decay as follow :

$$
h(n)=\sum_{i} \alpha_{i} \exp ^{-t / \tau_{i}}
$$

The amplitudes $\alpha_{i}$ and the lifetimes $\tau_{i}$ can be considered to be adjustable parameters. They are calculated employing Simplex algorithm minimizing the error of the equation :

$$
\left[y(n)-y^{\prime}(n)\right]=\varepsilon(n)
$$

It is known that quenching can occur by different mechanisms. Dynamic quenching occurs when the excited-state fluorophore is deactivated upon contact with other molecules in solutions due to physical collision Lakowicz (2006). In this case, the fluorophore returned to the ground-state during a diffusive encounter with the quencher. The molecules are not chemically modified in the process and can be shown by the Stern-Volmer equation 4 .

$$
\frac{I_{0}}{I_{Q}}=1+K_{S V}[Q]=1+k_{q} \tau_{Q}[Q]
$$

where $\mathrm{I}_{0}$ and $\mathrm{I}_{Q}$ are the fluorescence intensities in the absence and presence of quencher respectively, $\tau_{Q}$ is the fluorescence lifetime in the presence of metal, $K_{S V}$ is the Stern-Volmer quenching constant and $k_{q}$ is the bimolecular quenching constant.

160 If dynamic quenching takes place, the intensity of fluorescence and lifetime follows the relationship:

$$
\frac{I_{0}}{I_{Q}}=\frac{\tau_{0}}{\tau_{Q}}
$$

In this expression $\tau_{0}$ is the fluorescence lifetime in the absence of quencher. It is noteworthy that dynamic quenching is a time-dependent process.

Aside from collisional quenching, fluorescence quenching can occur due to a complex formation. Fluorophores can form non-fluorescent complexes with quenchers Lakowicz (2006). This process is referred to as static quenching since it occurs at ground-state and does not rely on diffusion or molecular collisions. 
The dependence of the fluorescence intensity upon quencher concentration is given by the following expression

$$
\frac{I_{0}}{I_{Q}}=1+K_{S}[Q]
$$

where $K_{S}$ is the association constant of the complex.

Note that the $I_{0} / I_{Q}$ on $[\mathrm{Q}]$ is linear, which is identical to dynamic quenching. Hence, the measurement of fluorescence lifetimes is the most ultimate method to distinguish static and dynamic quenching.

\section{Results \& Discussions}

175 3.1. Steady-State and fluorescence quenching results

The CP/PARAFAC decomposition of SA-Eu ${ }^{3+}$ quenching experiment shows that two components are obtained in this interaction (Fig. 1). The first component $\left(\lambda_{e x} / \lambda_{e m}\right)=295 / 410 \mathrm{~nm}$ shows a fluorescence intensity and is obviously the excitation/emission wavelength of SA Tauler et al. (1998) whereas, the second component does not show any fluorescence intensity variation (Fig. 2, A) nor fluorescence peak. Thus, the peak could be a noise factor and was not considered in the following discussion.

The relative contributions of each components of SA-Eu ${ }^{3+}$ interaction are shown in Fig. 2 A. indicating the quenching of SA depending on the $\mathrm{Eu}^{3+}$ concentration. The corresponding conditional stability constant ( $\mathrm{K}$ value) for the first component is reported in Table 1 and was estimated as $\log \mathrm{K}_{S A}=3.38$ based on the $1: 1$ complexation assumption between metal and ligand. This value is higher than $\log \mathrm{K}=2.08 \pm 0.02$ obtained by Aoyagi et al. (2004) at pH 4 from the slope of the intensity SternVolmer plot and higher than $\log K=2.02 \pm 0.05$

obtained by Hasegawa et al. (1989) at pH 6 from the glass electrode method. The stability constant of SA-Eu${ }^{3+}$ found in this study is consistent considering that the stability constant should increase when $\mathrm{pH}$ increases.

As for the extracted humic substances, the presence of abundant aromatic structures in organic matter provides reasonably good fluorescence characteristics. CP/PARAFAC decomposition of the EEMs obtained in the case of HA 
Table 1: Stability constants and average fluorescence lifetimes of SA and HSs complexed with $\underline{\mathrm{Eu}^{3+}}$

\begin{tabular}{ccccccc}
\hline & $\mathbf{L o g} \mathbf{K}_{S A}$ & $\mathbf{L o g} \mathbf{K}_{., C_{1}}$ & $\mathbf{L o g} \mathbf{K}_{., C_{2}}$ & $\mathbf{L o g} \mathbf{K}_{., C_{3}}$ & $\tau_{a}^{A} \mathbf{n s}$ & $\tau_{a}^{B} \mathbf{n s}$ \\
\hline $\mathbf{S A}$ & 3.38 & - & - & - & 2.97 & - \\
$\mathbf{H A}$ & - & 4.43 & 5.08 & 3.63 & 0.61 & 11.33 \\
$\mathbf{F A}$ & - & 4.42 & 4.43 & - & 0.73 & 12.54 \\
\hline
\end{tabular}

interaction with $\mathrm{Eu}^{3+}$ suggests the presence of three components (Fig. 1]B). The first component in the UV range is located at $\left(\lambda_{e x} / \lambda_{e m}\right)=270 / 445 \mathrm{~nm}$, and has been designated as peak A. It was attributed to humic substances Coble (1996). The relative contribution of this component to the EEMs during the quenching experiment is shown in Fig. 2.B. and the corresponding stability constant is $\log \mathrm{K}_{H A, C_{1}}=4.43$ (Table 1). The second component $\left(\lambda_{e x} / \lambda_{e m}\right)=270 / 505 \mathrm{~nm}$ shows a longer emission wavelength characteristic of a higher conjugated structure usually found in soils. This component behaves similarly to component 1 (Fig. 2,B) and has the higher stability constant $\left(\log \mathrm{K}_{H A, C_{2}}=5.08\right)$. The third component $\left(\lambda_{e x} / \lambda_{e m}\right)=250 / 395 \mathrm{~nm}$ is more relative to the protein-like structures and presents a weaker quenching effect. This means that the related fluorescent chemical functions are less affected by europium or less complexing. This is demonstrated by the lowest stability constant value $\log \mathrm{K}_{H A, C_{3}}=3.63$. In comparison, Wei et al. (2015) reported two stability constants using synchronous fluorescence spectroscopy. Their constants at $\mathrm{pH} 6$ are higher than found in our study : $\log K_{1}=5.51$ and $\log K_{2}=5.60$. However, the value reported by Hahn et al. (2017) using ultrafiltration and CE-ICP-MS at $\mathrm{pH}=5.00 \pm 0.05$ seems to be also higher than ours : $\log K=6.36 \pm 0.02$ and $\log K=6.1 \pm 0.1$. This work clearly shows that several fluorescent sites complex the europium in this particular HA from St. Lawrence estuary. More investigation should be done to compare the data, in particular for the $\mathrm{pH}$ conditions.

For the FA, two components were found by CP/PARAFAC showing an interesting fluorescence quenching (Fig. 2, C.) The first component (Fig. 1. C) 
located at $\left(\lambda_{e x} / \lambda_{e m}\right)=310 / 415 \mathrm{~nm}$ is generally another marine humic-like component and is designated as peak M Coble (1996). The second component $\left(\lambda_{e x} / \lambda_{e m}\right)=265 / 475 \mathrm{~nm}$ is larger and is designated as peak $\mathrm{A}$ Coble (1996) with a slight red shift in emission. The corresponding fluorescence intensities are given in Fig. 2. C. showing a homogeneous and similar behavior of both components. This is demonstrated by the values of the stability constants respectively $\log \mathrm{K}_{F A, C_{1}}=4.42$ and $\log \mathrm{K}_{F A, C_{2}}=4.43$ of the first and second component. The study of Chung et al. (2005) reported a stability constant at $\mathrm{pH}$ 5 equal to $6.4 \pm 0.2$ for $\mathrm{FA}-\mathrm{Eu}(\mathrm{III})$ interaction using synchronous fluorescence spectroscopy. This value is higher than our stability constant. This data is in good agreement with an other published work : $\log \mathrm{K}=6.00 \pm 0.10$ Bidoglio et al. 230 (1991). Furthermore, Lead et al. (1998) reported only one stability constant at a pH between 2.5 and $3.5: \log \mathrm{K}=2.36 \pm 0.13$ much like Wei et al. (2015) : $\log K=5.84$. The values of the stability constants found in the literature are higher due to the fact that the fulvic acids studied are mainly derived from soils or rivers and may have greater complexing capacities than the fulvic acids used in this study.

\subsection{TRLFS results}

As mentioned above in section 2.3 , the quenching process cannot be assessed using steady-state measurements. Therefore, fluorescence lifetime measurements using TRLFS are the most ultimate method to distinguish whether static, dynamic or both quenching processes occur based on Stern-Volmer plots Lakowicz (2006).

After S-S quenching experiments, eight samples out of twelve from the quenching curves were selected for lifetime measurement, because of the time required for each measure. The first sample does not contain any concentration of $\mathrm{Eu}^{3+}$ while the seven other samples contain an increasing volume of it at a concentration varying from $2.2 \times 10^{-5} \mathrm{M}$ to $2.2 \times 10^{-3} \mathrm{M}$.

For the interaction between $\mathrm{SA}-\mathrm{Eu}^{3+}$, the decay was found to be monoexponential. The average lifetime $\tau_{a}$ recorded was $2.97 \pm 0.2$ ns. In Fig. 3.A 

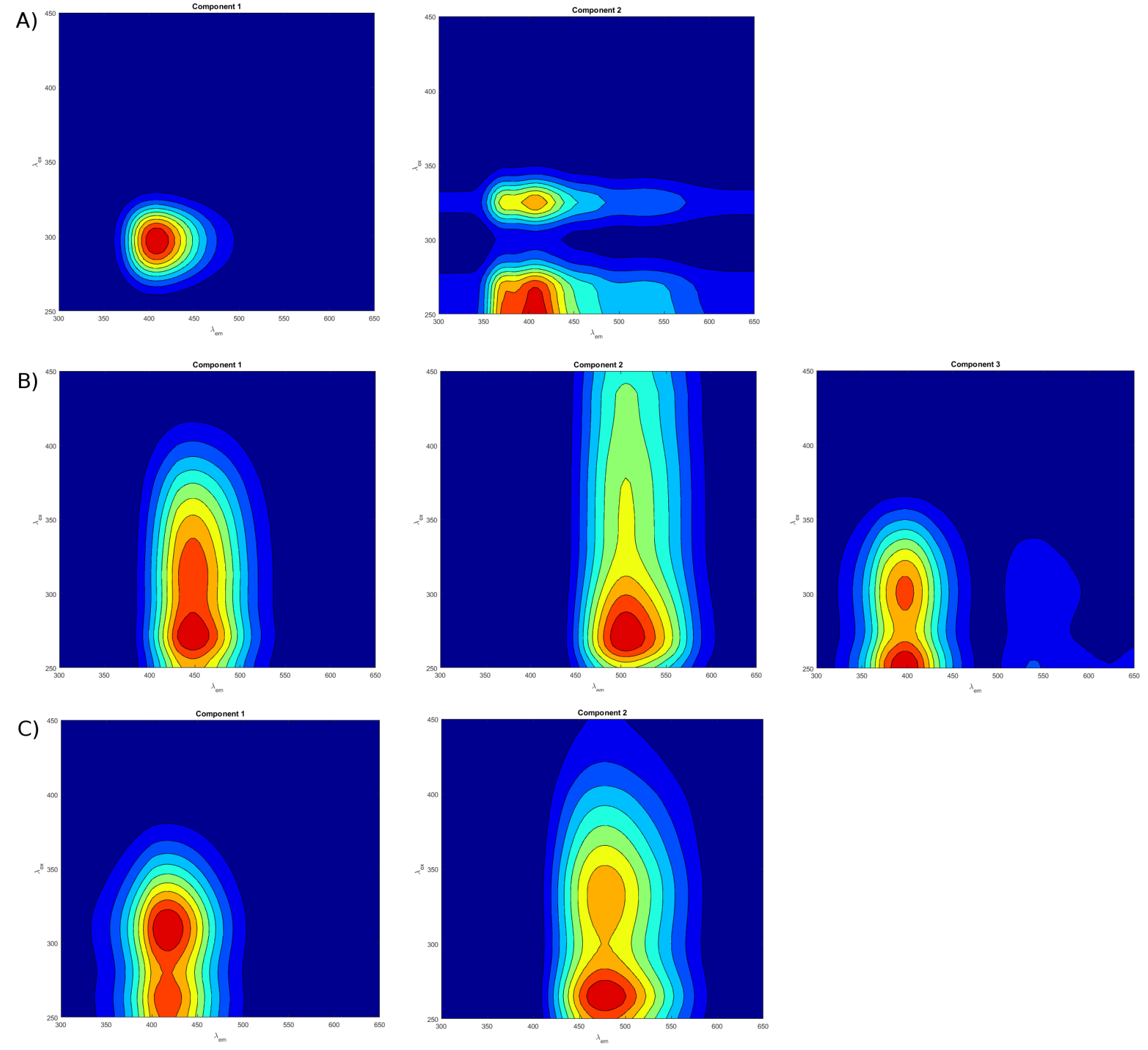

Figure 1: Components obtained for A) Salicylic acid- $-\mathrm{Eu}^{3+}$ B) Humic acid--Eu ${ }^{3+}$ and C) Fulvic acid-Eu ${ }^{3+}$ interaction after $\mathrm{CP} / \mathrm{PARAFAC}$ decomposition 

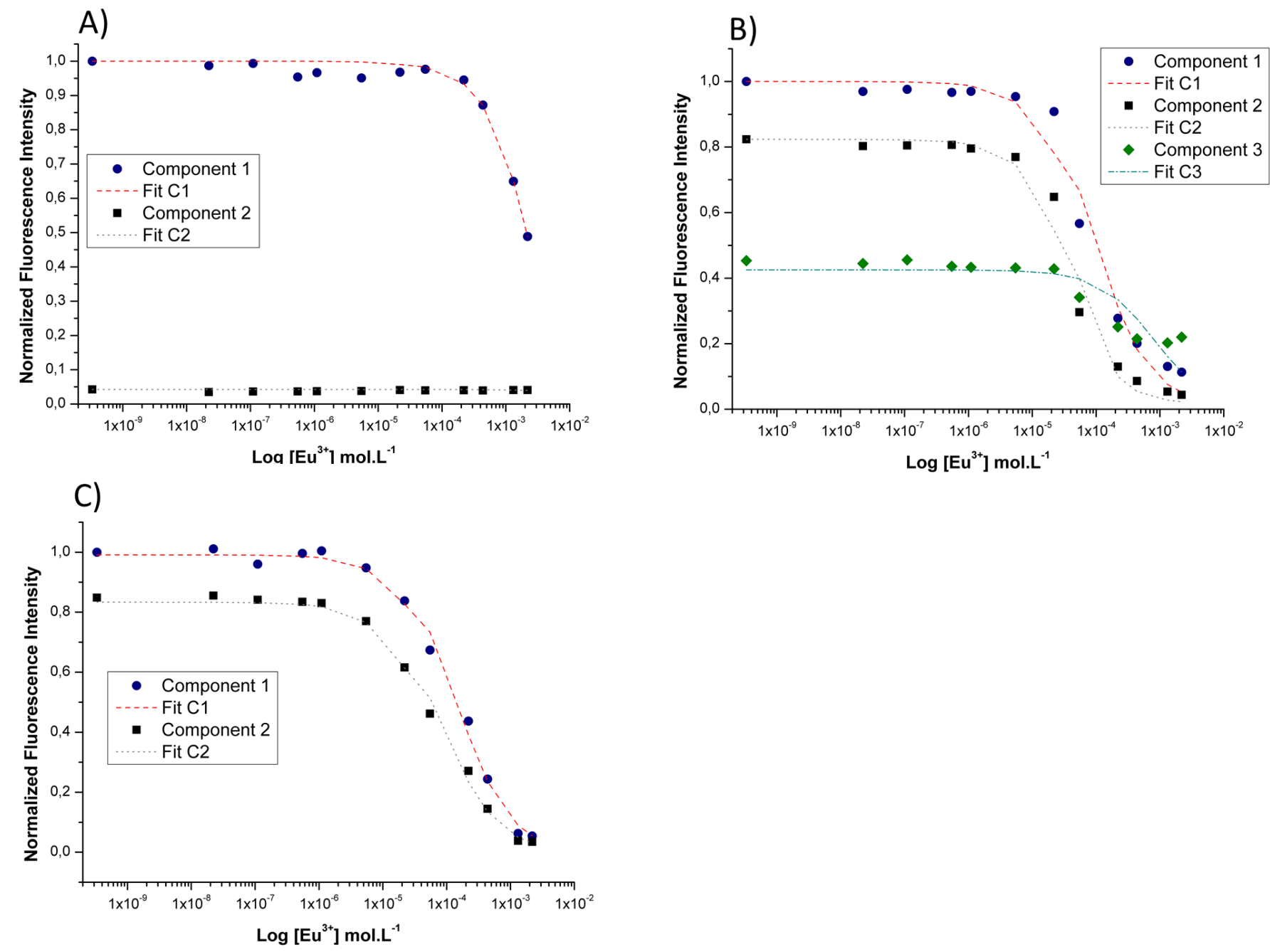

Figure 2: Variation of the CP/PARAFAC components contributions to the EEMs of A) Salicylic acid B) Humic acid and C) Fulvic acid as a function of $\mathrm{Eu}^{3+}$ concentration 
$I_{0} / I_{Q}$ and $\tau_{0} / \tau_{Q}$ are shown as a function of the concentration of $\mathrm{Eu}^{3+}$. The solid red line shows the linear regression of the Stern-Volmer plot intensity for $I_{0} / I_{Q}$ and $\tau_{0} / \tau_{Q}$. The value of the lifetime ratio seems to be dependent on metal addition. This indicates that the observed quenching involves a dynamic quenching process or a mix of static and dynamic quenching, as the $I_{0} / I_{Q}$ and $\tau_{0} / \tau_{Q}$ should have the same slope in case of pure dynamic process Lakowicz (2006). On the other hand, Aoyagi et al. (2004) found a fluorescence lifetime equal to $2.02 \pm 0.01 \mathrm{~ns}$ and a static quenching occurring for SA-Eu${ }^{3+}$ interaction. This discrepancy could be due to the $\mathrm{pH}$ value as they worked at $\mathrm{pH} 4$.

For $\mathrm{HA}-\mathrm{Eu}^{3+}$ interaction, CP/PARAFAC decomposition gives three components while the time deconvolution suggests a bi-exponential decay and two lifetimes denoted by exponent indexes $\tau^{A}$ and $\tau^{B}$. One very short, indicated by $\tau_{0}^{A} / \tau_{Q}^{A}$ in Fig. 3. B and the second one, specified by $\tau_{0}^{B} / \tau_{Q}^{B}$, longer than the first one (11.33 ns). The two fluorescence lifetimes turned out to be independent on total concentration of $\mathrm{Eu}^{3+}$. This suggests a static quenching. The dependence of $I_{0} / I_{Q}$ of component 1 and component 2 on $\left[\mathrm{Eu}^{3+}\right]$ is therefore non-linear. In the case of static and dynamic quenching, the characteristic feature of the Stern-Volmer plot is an upward curvature, concave towards the y-axis. The concave pattern of the Stern-Volmer plot is interpreted as the manifestation of fluorophores that are not accessible for quenching Lakowicz (2006). Thus, the data cannot be accurately fitted using the linear Stern-Volmer equation 4 in this situation. However, Reiller and Brevet (2010) observed a bi-exponential decay for the luminescence of $\mathrm{Eu}(\mathrm{III})-\mathrm{HSs}$ complexes and reported a deactivation of at least two different excited-states from $\mathrm{Eu}$ (III) site. The reported values of fluorescence lifetime cannot be compared to our study since they measured the fluorescence lifetime values from $\mathrm{Eu}(\mathrm{III})$ site.

275

The intensity ratios of the fulvic acid with $\mathrm{Eu}^{3+}$ in Fig. 3 . C. seem to be linear except the penultimate point which could be attributed to an error in the measurement. According to Tiseanu et al. (1998), the existence of a tri-modal decay time distribution with time values centered around $0.7,3$ and 11 ns was 
A)

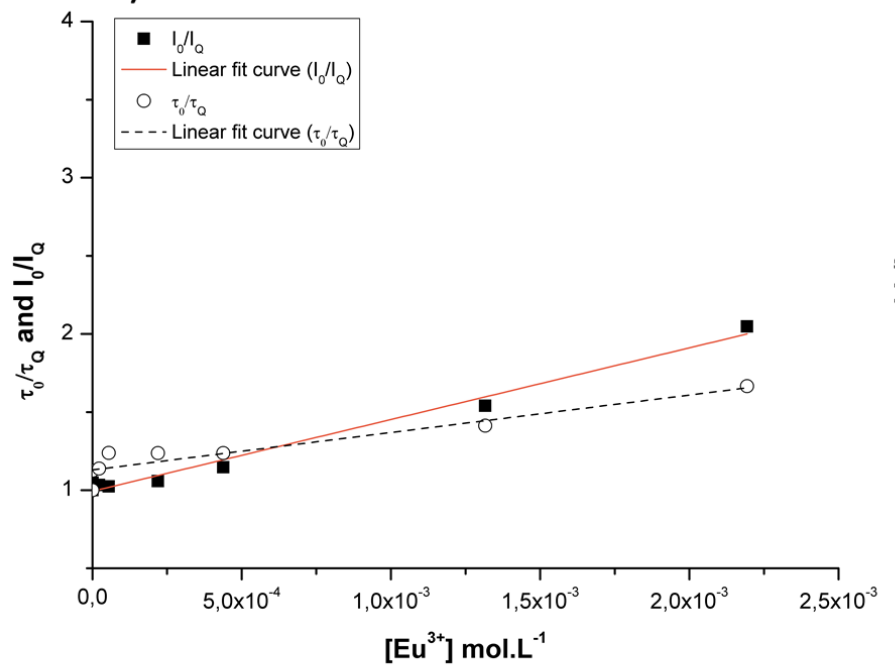

B)

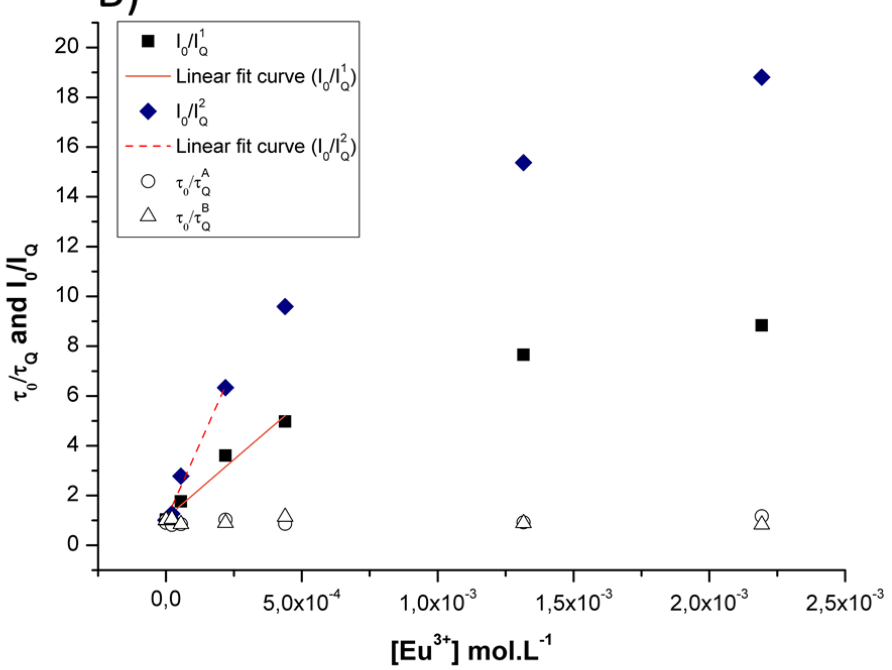

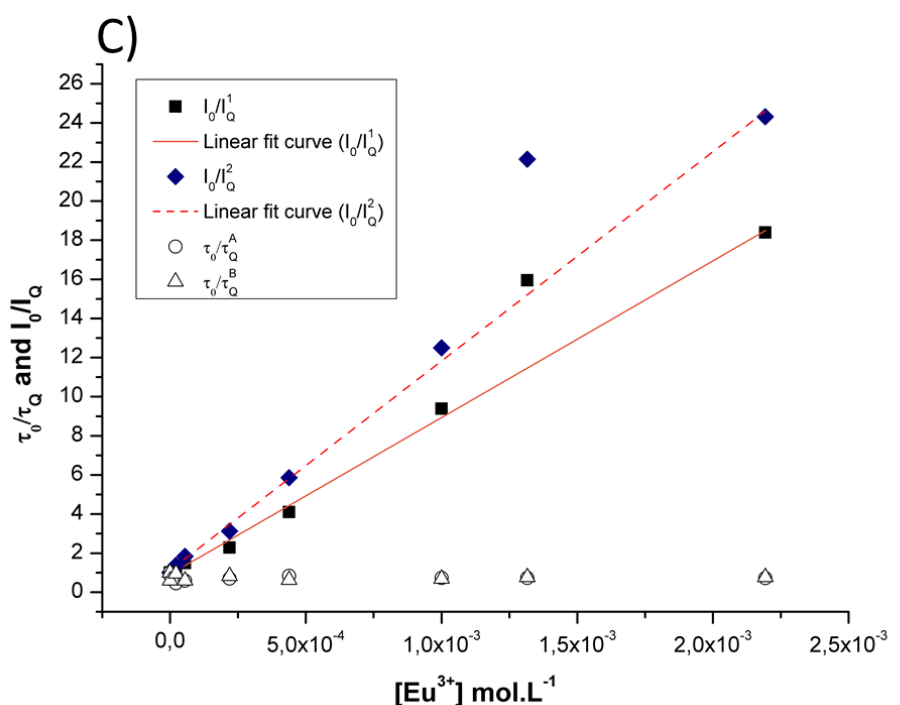

Figure 3: Stern-Volmer plot showing the intensities ratio and the fluorescence lifetimes ratio of A) Salicylic acid-Eu ${ }^{3+}$, B) Humic acid-Eu ${ }^{3+}$ and C) Fulvic acid-Eu ${ }^{3+}$ interactions 

interactions studied were calculated using the one-site $1: 1$ model and are in concordance with literature for SA and HA. For FA, we found constants slightly 
lower than those in the literature which may be due to the origin of FA. It is

noteworthy that TRLFS successfully discriminates the fluorescence quenching process that takes place and gives original lifetimes of HSs and constant of complexation with $\mathrm{Eu}(\mathrm{III})$. Further application can be extended to quantitatively analyze other lanthanides and actinides.

\section{References}

315 Aoyagi N, Toraishi T, Geipel G, Hotokezaka H, Nagasaki S, Tanaka S. Fluorescence characteristics of complex formation of Europium (III)-Salicylate. Radiochimica Acta 2004;92(9-11):589-93.

Bidoglio G, Grenthe I, Qi P, Robouch P, Omentto N. Complexation of Eu and $\mathrm{Tb}$ with fulvic acids as studied by time-resolved laser-induced fluorescence. Talanta 1991;38(9):999-1008.

Brevet J, Claret F, Reiller PE. Spectral and temporal luminescent properties of $\mathrm{Eu}$ (III) in humic substance solutions from different origins. Spectrochimica Acta Part A: Molecular and Biomolecular Spectroscopy 2009;74(2):446-53.

Bro R. Parafac. tutorial and applications. Chemometrics and Intelligent Laboratory Systems 1997;38(2):149-71.

Choppin G. Actinide speciation in the environment. Journal of Radioanalytical and Nuclear Chemistry 2007;273(3):695-703.

Chung K, Lee W, Cho Y, Choi G, Lee C. Comparison of synchronous and laser-induced fluorescence spectroscopy applied to the $\mathrm{Eu}$ (III)-fulvate complexation. Talanta 2005;65(2):389-95.

Cobelo-García A, Filella M, Croot P, Frazzoli C, Du Laing G, Ospina-Alvarez N, Rauch S, Salaun P, Schäfer J, Zimmermann S. COST action TD1407: Network on technology-critical elements (NOTICE)from environmental processes to human health threats. Environmental Science and Pollution Research 2015;22(19):15188-94. 
Coble PG. Characterization of marine and terrestrial DOM in seawater using excitation-emission matrix spectroscopy. Marine chemistry 1996;51(4):32546.

Collins RN, Saito T, Aoyagi N, Payne TE, Kimura T, Waite TD. Applications of time-resolved laser fluorescence spectroscopy to the environmental biogeochemistry of actinides. Journal of Environmental Quality 2011;40(3):731-41.

Czerwinski K, Kim J, Rhee D, Buckau G. Complexation of trivalent actinide ions $\left(\mathrm{Am}^{3+}, \mathrm{Cm}^{3+}\right)$ with humic acid: the effect of ionic strength. Radiochimica Acta 1996;72(4):179-88.

Dube A, Zbytniewski R, Kowalkowski T, Cukrowska E, Buszewski B. Adsorption and migration of heavy metals in soil. Polish journal of environmental studies 2001;10(1):1-10.

Hahn R, Hein C, Sander JM, Kautenburger R. Complexation of europium and uranium with natural organic matter (NOM) in highly saline water matrices analysed by ultrafiltration and inductively coupled plasma mass spectrometry (ICP-MS). Applied Geochemistry 2017;78:241-9.

Hasegawa Y, Morita Y, Hase M, Nagata M. Complexation of lanthanoid (III) with substituted benzoic or phenylacetic acids and extraction of these acids. Bulletin of the Chemical Society of Japan 1989;62(5):1486-91.

Hasegawa Y, Yamazaki N, Usui S, Choppin GR. Effects of phenyl groups on thermodynamic parameters of lanthanoid (III) complexation with aromatic carboxylic acids. Bulletin of the Chemical Society of Japan 1990;63(8):216972 .

Hiraide M. Heavy metals complexed with humic substances in fresh water. Analytical Sciences 1992;8(4):453-9.

Irving H, Sinha S. The formation constants of europium (III)-salicylate complexes and their extraction into isoamyl alcohol. Analytica Chimica Acta 1970;49(3):449-54. 
Jain A, Yadav K, Mohapatra M, Godbole S, Tomar B. Spectroscopic investigation on europium complexation with humic acid and its model compounds. Spectrochimica Acta Part A: Molecular and Biomolecular Spectroscopy $2009 ; 72(5): 1122-6$.

Kim J, Buckau G, Bryant E, Klenze R. Complexation of americium (III) with humic acid. Radiochimica Acta 1989;48(3-4):135-44.

Kim J, Rhee D, Wimmer H, Buckau G, Klenze R. Complexation of trivalent actinide ions $\left(\mathrm{Am}^{3+}, \mathrm{Cm}^{3+}\right)$ with humic acid: a comparison of different experimental methods. Radiochimica Acta 1993;62(1-2):35-44.

Kuke S, Marmodee B, Eidner S, Schilde U, Kumke MU. Intramolecular deactivation processes in complexes of salicylic acid or glycolic acid with $\mathrm{Eu}$ 375 (III). Spectrochimica Acta Part A: Molecular and Biomolecular Spectroscopy 2010;75(4):1333-40.

Kumke M, Tiseanu C, Abbt-Braun G, Frimmel F. Fluorescence decay of natural organic matter (NOM)influence of fractionation, oxidation, and metal ion complexation. Journal of Fluorescence 1998;8(4):309-18.

${ }_{380}$ Lakowicz JR. Principles of fluorescence spectroscopy. Springer Science and Business Media, 2006.

Lead J, Hamilton-Taylor J, Peters A, Reiner S, Tipping E. Europium binding by fulvic acids. Analytica Chimica Acta 1998;369(1):171-80.

Luciani X, Mounier S, Redon R, Bois A. A simple correction method of inner filter effects affecting FEEM and its application to the PARAFAC decomposition. Chemometrics and Intelligent Laboratory Systems 2009;96(2):227-38.

Lukman S, Saito T, Aoyagi N, Kimura T, Nagasaki S. Speciation of Eu ${ }^{3+}$ bound to humic substances by time-resolved laser fluorescence spectroscopy (TRLFS) and parallel factor analysis (PARAFAC). Geochimica et Cosmochimica Acta 2012;88:199-215. 
Monsallier JM, Artinger R, Denecke MA, Scherbaum FJ, Buckau G, Kim JI. Spectroscopic study (TRLFS and EXAFS) of the kinetics of An (III)/Ln (III) humate interaction. Radiochimica Acta 2003;91(10):567-74.

Morgenstern M, Klenze R, Kim J. The formation of mixed-hydroxo complexes of $\mathrm{Cm}$ (III) and Am III) with humic acid in the neutral $\mathrm{pH}$ range. Radiochimica Acta 2000;88(1):7-17.

Moulin C, Decambox P, Mauchien P, Moulin V, Theyssier M. On the use of laser-induced time-resolved spectrofluorometry for interaction studies between organic matter and actinides: application to curium. Radiochimica Acta 1991;52(1):119-26.

Pacold JI, Tatum DS, Seidler GT, Raymond KN, Zhang X, Stickrath AB, Mortensen DR. Direct observation of $4 \mathrm{f}$ intrashell excitation in luminescent eu complexes by time-resolved X-ray absorption near edge spectroscopy. Journal of the American Chemical Society 2014;136(11):4186-91.

Panak P, Klenze R, Kim J. A study of ternary complexes of Cm (III) with humic acid and hydroxide or carbonate in neutral $\mathrm{pH}$ range by time-resolved laser fluorescence spectroscopy. Radiochimica Acta 1996;74(s1):141-6.

Reiller PE, Brevet J. Bi-exponential decay of Eu (III) complexed by suwannee river humic substances: spectroscopic evidence of two different excited species. Spectrochimica Acta Part A: Molecular and Biomolecular Spectroscopy 2010;75(2):629-36.

Ryan DK, Weber JH. Copper (II) complexing capacities of natural waters by fluorescence quenching. Environmental Science and Technology 1982;16(12):866-72.

${ }_{415}$ Sachs S, Bernhard G. Influence of humic acids on the actinide migration in the environment: suitable humic acid model substances and their application in studies with uranium-A review. Journal of Radioanalytical and Nuclear Chemistry 2011;290(1):17-29. 
Saito T, Aoyagi N, Terashima M. Europium binding to humic substances extracted from deep underground sedimentary groundwater studied by time-resolved laser fluorescence spectroscopy. Journal of Nuclear Science and Technology 2017;54(4):444-51.

Schmeide K, Reich T, Sachs S, Bernhard G. Plutonium (III) complexation by humic substances studied by X-ray absorption fine structure spectroscopy. Inorganica Chimica Acta 2006;359(1):237-42.

Stevenson FJ. Humus chemistry: genesis, composition, reactions. John Wiley and Sons, 1994.

Swift R. Method for extraction of IHSS soil fulvic and humic acids. Soil Science Society of America and American Society of Agronomy 1996;:1011-69.

Tauler R, Marqués I, Casassas E. Multivariate curve resolution applied to three-way trilinear data: Study of a spectrofluorimetric acid-base titration of salicylic acid at three excitation wavelengths. Journal of Chemometrics 1998;12(1):55-75.

Tiseanu CD, Kumke M, Frimmel F, Klenze R, Kim J. Time-resolved fluorescence spectroscopy of fulvic acid and fulvic acid complexed with $\mathrm{Eu}^{3+}$ : A comparative study. Journal Of Photochemistry and Photobiology A: Chemistry 1998;117(3):175-84.

Toraishi T, Nagasaki S, Tanaka S. A theoretical study on molecular structure of Eu (III)-salicylate complexes in aqueous system. Journal of Molecular Structure: THEOCHEM 2005;757(1):87-97.

Tremblay L, Gagné JP. Organic matter distribution and reactivity in the waters of a large estuarine system. Marine Chemistry 2009;116(1):1-12.

Wei J, Han L, Song J, Chen M. Evaluation of the interactions between water extractable soil organic matter and metal cations (Cu (II), Eu (III)) using excitation-emission matrix combined with parallel factor analysis. International Journal of Molecular Sciences 2015;16(7):14464-76. 
Zepp RG, Sheldon WM, Moran MA. Dissolved organic fluorophores in southeastern US coastal waters: correction method for eliminating rayleigh and raman scattering peaks in excitation-emission matrices. Marine Chemistry 2004;89(1):15-36. 